\title{
Twinning partial multiplication at grain boundary in nanocrystalline fcc metals
}

\author{
Y. T. Zhu, ${ }^{1, a)}$ X. L. Wu, ${ }^{2}$ X. Z. Liao ${ }^{3}$ J. Narayan, ${ }^{1}$ S. N. Mathaudhu, ${ }^{4}$ and L. J. Kecskés ${ }^{4}$ \\ ${ }^{1}$ Department of Materials Science and Engineering, North Carolina State University, Raleigh, \\ North Carolina 27695, USA \\ ${ }^{2}$ State Key Laboratory of Nonlinear Mechanics, Institute of Mechanics, Chinese Academy of Sciences, \\ Beijing 100080, People's Republic of China \\ ${ }^{3}$ School of Aerospace, Mechanical, and Mechatronic Engineering, The University of Sydney, \\ New South Wales 2006, Australia \\ ${ }^{4}$ U.S. Army Research Laboratory, Aberdeen Proving Ground, Maryland 21005, USA
}

(Received 11 May 2009; accepted 6 July 2009; published online 22 July 2009)

\begin{abstract}
Most deformation twins in nanocrystalline face-centered cubic (fcc) metals have been observed to form from grain boundaries. The growth of such twins requires the emission of Shockley partials from the grain boundary on successive slip planes. However, it is statistically improbable for a partial to exist on every slip plane. Here we propose a dislocation reaction and cross-slip mechanism on the grain boundary that would supply a partial on every successive slip plane for twin growth. This mechanism can also produce a twin with macrostrain smaller than that caused by a conventional twin. (c) 2009 American Institute of Physics. [DOI: 10.1063/1.3187539]
\end{abstract}

Twinning is a major deformation mode in metals. ${ }^{1,2}$ Nanocrystalline (NC) face-centered cubic (fcc) metals and alloys with medium-to-high stacking-fault energy have been observed to deform by twinning more easily than their coarse-grained counterparts. ${ }^{3-12}$ In addition, contrary to coarse-grained metals, which become more difficult to deform by twinning with decreasing grain size, $\mathrm{NC}$ fcc metals may be first easier to deform by twinning with decreasing grain size, and then become more difficult again, i.e., an inverse grain-size effect on twinning. ${ }^{13}$

Deformation twins usually form in $\mathrm{NC}$ fcc metals via mechanisms different from those proposed for coarsegrained materials. ${ }^{2}$ Several twinning mechanisms have been proposed for and observed in NC fcc metals, including the coincidental overlapping of wide stacking fault ribbons, ${ }^{14,15}$ partial emissions from grain boundaries, ${ }^{2,5,6,11,14-18}$ grain boundary splitting and migration, ${ }^{15,16}$ random activation of partials (RAP), ${ }^{2}$ dislocation cross-slip mechanism, ${ }^{19}$ selfpartial multiplication mechanisms, ${ }^{20-22}$ and dislocation rebound mechanism. ${ }^{22}$

Single twins with two coherent boundaries are observed most frequently under high-resolution electron microscopy (HREM) and by molecular dynamics simulations. ${ }^{2,5,6,11,14-18}$ If a single twin lamellar extends across a grain, it could be formed by the RAP mechanism, ${ }^{2}$ the dislocation rebound mechanism, ${ }^{22}$ or the partial emissions from grain boundary. ${ }^{6}$ However, if a single twin grows from a grain boundary but terminates inside a grain (see Fig. 1), it can only be formed by the partial emissions from grain boundary. In this case, Shockley partials need to be emitted from the grain boundary on the left on successive slip planes. It has been reported that partial dislocations can be readily available and emit from nonequilibrium grain boundary to nucleate a deformation twin., ${ }^{4,23}$ However, it is statistically and practically impossible for a partial dislocation to exist on every slip plane to grow a single twin. Nucleation of a new partial on every slip

\footnotetext{
a) Author to whom correspondence should be addressed. Electronic mail: ytzhu@ncsu.edu.
}

plane is difficult because of the required high energy. It has been a long-standing puzzle on the origin of the twinning partials that lead to the formation of a single twin from grain boundary.

Here we propose two mechanisms that can supply a twinning partial on every slip plane at the grain boundary to grow a single twin continuously. The first mechanism involves the emission of partials with the same Burgers vector, which produces a conventional twin with expected large shear strain. ${ }^{2}$ The second mechanism involves the emission of partials with two different Burgers vectors, which produce a single twin with reduced shear strain. The details are described below.

We start with the first mechanism. Figure 2 illustrates a square grain with a dislocation line parallel to the grain boundary. The sense (direction) of the dislocation line is $\boldsymbol{\xi}$ $=1 / \sqrt{6}[112]$, and its Burgers vector is $\mathbf{b}=1 / 2[101]$. The square is in the $(11 \overline{1})$ slip plane. Under an applied stress, this perfect dislocation dissociates into two partials,

$$
\mathbf{b} \rightarrow \mathbf{b}_{1}+\mathbf{b}_{2},
$$

where $\mathbf{b}_{1}=1 / 6[112]$ is parallel to the dislocation line, while $\mathbf{b}_{2}=1 / 6[2 \overline{1} 1]$ has a $60^{\circ}$ angle with it.

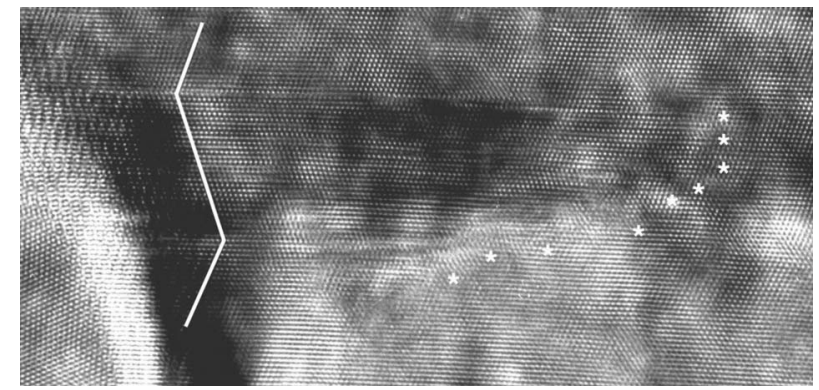

FIG. 1. A HREM image of a twin formed by plastically deforming electrodeposited NC Ni. The twin was formed by the emission of partials from the grain boundary on the left and it ended in the grain interior as marked by the white asterisks. 


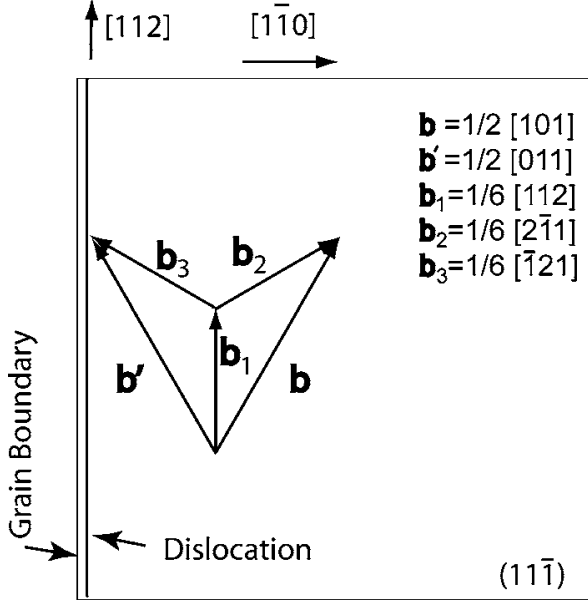

FIG. 2. Schematics of a square grain with a dislocation line parallel to the grain boundary. The dislocation has a Burgers vector of $\mathbf{b}=1 / 2[101]$. The slip plane is $(11 \overline{1})$, which is parallel to the page.

Under an applied shear stress with appropriate orientation, the partial $\mathbf{b}_{2}$ glides on the (11) $)$ plane across the grain to form a stacking fault. Partial $\mathbf{b}_{\mathbf{1}}$ is a pure screw partial and cross slips to the next $(11 \overline{1})$ plane, where it undergoes the following reaction,

$$
\mathbf{b}_{1} \rightarrow \mathbf{b}+\left(-\mathbf{b}_{2}\right)
$$

For this reaction, we envision that atoms in the core of $\mathbf{b}_{\mathbf{1}}$ shuffle under applied stress to simultaneously form $-\mathbf{b}_{\mathbf{2}}$ and $\mathbf{b}$, separated by a distance equal to the core width of $\mathbf{b}_{\mathbf{1}}$, $a / \sqrt{6}$. Atomic shuffling has been observed in the process of dislocation emission from grain boundaries. ${ }^{7}$ At this distance, the attractive force between $-\mathbf{b}_{2}$ and $\mathbf{b}$ per unit dislocation line length can be derived as $F=\sqrt{6} G b / 8 \pi$, where $G$ is the shear modulus, and $b$ is the magnitude of the perfect dislocation. ${ }^{24}$ The resolved shear stress that is required to drive $\mathbf{b}$ away from $-\mathbf{b}_{2}$ can be described by $\tau=F / b$ $=\sqrt{6} G / 8 \pi \approx G / 10$. This stress level is not out of reach by local stress concentrations plus applied shear stress. For example, the stress concentration near a stacking fault in $\mathrm{Cu}$ could reach $3.5 \mathrm{GPa}^{17}$ In addition, fluctuation of thermal energies at the grain boundary could also help with the reaction. Therefore, the reaction described in Eq. (2) is plausible.

After the reaction (2), $-\mathbf{b}_{\mathbf{2}}$ stays at the grain boundary and $\mathbf{b}$ is driven toward the grain interior by the applied stress. The applied stress also makes $\mathbf{b}$ dissociate into $\mathbf{b}_{\mathbf{1}}$ and $\mathbf{b}_{2}$ according to Eq. (1), forming a wide stacking fault ribbon. ${ }^{25} \mathbf{b}_{2}$ would be the leading partial because the applied stress favors its slip. The slip of $\mathbf{b}_{\mathbf{2}}$ nucleates a single twin, and $\mathbf{b}_{1}$ returns to the grain boundary due to insufficient resolved shear stress to drive it across the grain.

Repetition of the above dislocation reaction and cross slip enables the single twin to grow continuously. Such a twin growth process leaves a partial $-\mathbf{b}_{2}$ on the left grain boundary and another partial $\mathbf{b}_{\mathbf{2}}$ on the right grain boundary on every slip plane in the twin. This changes the grain shape as shown in Fig. 3, as viewed in the $\mathbf{b}^{\prime}=[011]$ direction, which is perpendicular to $\mathbf{b}_{\mathbf{2}}$.

In the above description of the model, we specified the dislocation type and Burgers vector so that $\mathbf{b}_{\mathbf{1}}$ is a pure screw partial, making it easy for $\mathbf{b}_{\mathbf{1}}$ to cross slip into the next (11 $\left.\overline{1}\right)$

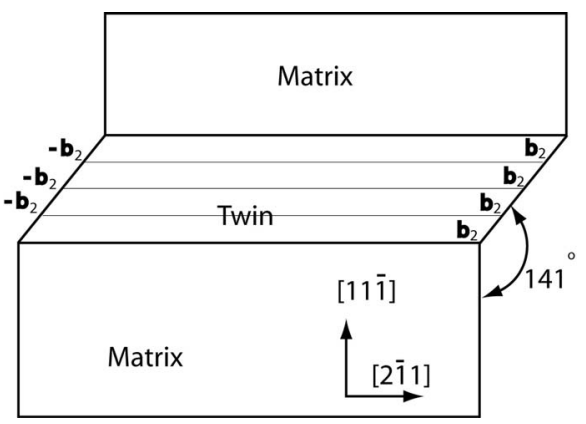

FIG. 3. The grain shape change caused by the first twinning mechanism in which all twinning partials have the same Burgers vector. The $141^{\circ}$ grain boundary kink can be seen from an orientation perpendicular to the Burgers vector.

plane. This is the easiest path for the twin growth. However, even if $\mathbf{b}_{\mathbf{1}}$ is a pure edge or a mixed type, it can still move to the next $(11 \overline{1})$ slip plane by climbing or climbing plus cross slip, although higher applied stress is needed for such a process. Note also that the dislocation climbing on the grain boundary should be much easier than in the grain interior because of higher diffusion rate and possibly also higher local residual stress.

Next we will describe the second mechanism, which produces less macroscopic strain than a conventional twin. As shown in Fig. 2, after cross slip, the partial $\mathbf{b}_{1}$ under appropriate applied stress can have another dislocation reaction,

$$
\mathbf{b}_{1} \rightarrow \mathbf{b}^{\prime}+\left(-\mathbf{b}_{3}\right),
$$

where $\mathbf{b}_{3}=1 / 6[\overline{1} 21]$ and $\mathbf{b}^{\prime}=1 / 2[011]$. The partial $-\mathbf{b}_{3}$ stays at the grain boundary and the $\mathbf{b}^{\prime}$ dissociates into $\mathbf{b}_{\mathbf{1}}$ and a new partial $\mathbf{b}_{3}$ (see Fig. 3). The applied shear stress drives the new partial $\mathbf{b}_{\mathbf{3}}$ away from the grain boundary to glide on the $(11 \overline{1})$ plane across the grain, which grows the twin.

If the reactions described in Eqs. (2) and (3) occur on alternative slip planes or occur for about the same number of times, the grain will change its shape along the $\mathbf{b}_{\mathbf{1}}$ direction (see Fig. 1), but the macroscopic strain will only be half of that shown in Fig. 3. This is because the slip of the partial $\mathbf{b}_{2}$ produces a strain of $a / \sqrt{6}$ along the $\mathbf{b}_{2}$ direction, while the slip of the partial $\mathbf{b}_{\mathbf{3}}$ produces a strain of $a / \sqrt{6}$ along the $\mathbf{b}_{\mathbf{3}}$ direction. From Fig. 2 we have the following relationship $\mathbf{b}_{2}+\mathbf{b}_{3}=\mathbf{b}_{1}$. Therefore, the total strain in every two slip planes is $a / \sqrt{6}$ along the $\mathbf{b}_{1}$ direction, producing a change in grain shape as shown in Fig. 4 when viewed from the $[1 \overline{1} 0]$ orientation.

In an HREM micrograph taken from a $\langle 110\rangle$ direction on the coherent twin boundary, the atomic-scale image has mirror symmetry across the twin boundary. If the HREM image shows a twinned grain with a $141^{\circ}$ grain boundary kink as shown in Fig. 3, then the twin was formed by the first mechanism, in which only one type of twinning partial (e.g., $\mathbf{b}_{2}$ ) was involved in producing the twin. On the other hand, if the HREM image shows a twinned grain with a $158^{\circ}$ grain boundary kink as shown in Fig. 4, it is not possible to determine the twinning mechanism. The reason can be explained with the help of Fig. 2.

As shown in Fig. 2, if a twin is produced by $\mathbf{b}_{2}$ partials (the first mechanism), the grain shape will be changed in the 


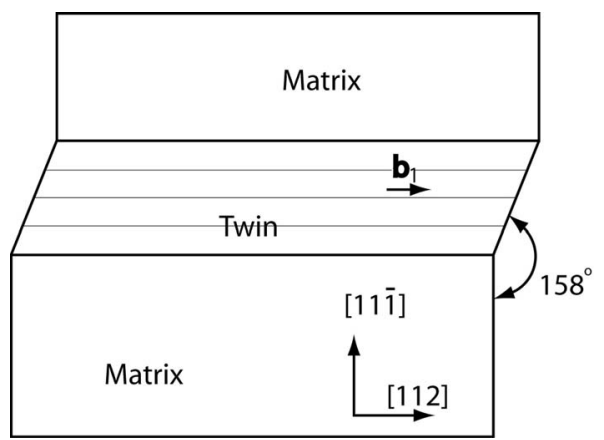

FIG. 4. The grain shape change caused by the second twinning mechanism in which approximately equal numbers of $\mathbf{b}_{2}$ partials and $\mathbf{b}_{3}$ partials are involved. The $158^{\circ}$ grain boundary kink can be seen from an orientation perpendicular to $\mathbf{b}_{\mathbf{1}}$.

$\mathbf{b}_{2}$ direction. The change in grain shape can be best viewed from an orientation that is perpendicular to $\mathbf{b}_{\mathbf{2}}$, which in this case is $\mathbf{b}^{\prime}$, producing the grain shape change with a $141^{\circ}$ grain boundary kink (Fig. 3). However, if viewed from the orientation $\mathbf{b}$, which has a $30^{\circ}$ angle to $\mathbf{b}_{2}$, the macroscopic strain will appear to be half of the value of the real macroscopic strain. In other words, the shape change of the grain will appear to be the same as that shown in Fig. 4, with a $158^{\circ}$ grain boundary kink, which is identical to the twin produced by the second mechanism. Therefore, the HREM image will not be able to differentiate the twinning mechanism in this case.

Following the above logic, a twin produced by the second mechanism will show either $158^{\circ}$ grain boundary kink if viewed from the [1 $1 \overline{1} 0]$ orientation, or $169^{\circ}$ grain boundary kink if viewed from the $\mathbf{b}$ or $\mathbf{b}^{\prime}$ orientation. Note that it is also possible to form a twin by the mixture of the first and the second mechanisms. This could happen when the twined grain rotates during the twinning process, which changes the stress orientation.

In practice, the grain boundary kink in a HREM micrograph is sharp enough for angle measurement only when the grain boundary is close to the edge-on orientation, which does not happen very often statistically. This makes the kinkangle measurement a tedious task. For example, the grain boundary kink in Fig. 1 is not obvious because grain boundary is far from the edge-on condition.
In summary, we have proposed two dislocation reaction and cross-slip mechanisms for Shockley twinning partials to multiply at grain boundaries in NC fcc metals. The proposed mechanisms solve a long-standing puzzle: what is the origin of Shockley partials for growing a twin from the grain boundary? Future work using molecular dynamics simulations to investigate the stresses in these mechanisms will shed more light into twin nucleation and growth in $\mathrm{NC}$ materials.

${ }^{1}$ J. W. Christian and S. Mahajan, Prog. Mater. Sci. 39, 1 (1995).

${ }^{2}$ X. L. Wu, X. Z. Liao, S. G. Srinivasan, F. Zhou, E. J. Lavernia, R. Z. Valiev, and Y. T. Zhu, Phys. Rev. Lett. 100, 095701 (2008).

${ }^{3}$ M. W. Chen, E. Ma, K. J. Hemker, H. W. Sheng, Y. M. Wang, and X. M. Cheng, Science 300, 1275 (2003).

${ }^{4}$ X. Z. Liao, Y. H. Zhao, Y. T. Zhu, R. Z. Valiev, and D. V. Gunderov, J. Appl. Phys. 96, 636 (2004).

${ }^{5}$ X. Z. Liao, Y. H. Zhao, S. G. Srinivasan, Y. T. Zhu, R. Z. Valiev, and D. V. Gunderov, Appl. Phys. Lett. 84, 592 (2004).

${ }^{6}$ V. Yamakov, D. Wolf, S. R. Phillpot, A. K. Mukherjee, and H. Gleiter, Nature Mater. 1, 45 (2002).

${ }^{7}$ H. Van Swygenhoven, P. M. Derlet, and A. G. Froseth, Nature Mater. 3, 399 (2004).

${ }^{8}$ Y. T. Zhu, X. Z. Liao, S. G. Srinivasan, and E. J. Lavernia, J. Appl. Phys. 98, 034319 (2005).

${ }^{9}$ Y. T. Zhu, X. Z. Liao, and R. Z. Valiev, Appl. Phys. Lett. 86, 103112 (2005).

${ }^{10}$ X. L. Wu, Y. T. Zhu, M. W. Chen, and E. Ma, Scr. Mater. 54, 1685 (2006)

${ }^{11}$ X. Z. Liao, F. Zhou, E. J. Lavernia, D. W. He, and Y. T. Zhu, Appl. Phys. Lett. 83, 5062 (2003).

${ }^{12}$ H. Rösner, J. Markmann, and J. Weissmüller, Philos. Mag. Lett. 84, 321 (2004).

${ }^{13}$ X. L. Wu and Y. T. Zhu, Phys. Rev. Lett. 101, 025503 (2008).

${ }^{14}$ X. Z. Liao, F. Zhou, E. J. Lavernia, S. G. Srinivasan, M. I. Baskes, D. W. He, and Y. T. Zhu, Appl. Phys. Lett. 83, 632 (2003).

${ }^{15}$ V. Yamakov, D. Wolf, S. R. Phillpot, and H. Gleiter, Acta Mater. 50, 5005 (2002).

${ }^{16}$ V. Yamakov, D. Wolf, S. R. Phillpot, A. K. Mukherjee, and H. Gleiter, Nature Mater. 3, 43 (2004)

${ }^{17}$ J. Wang and H. C. Huang, Appl. Phys. Lett. 85, 5983 (2004)

${ }^{18}$ X. L. Wu and Y. T. Zhu, Appl. Phys. Lett. 89, 031922 (2006).

${ }^{19}$ H. Fujita and T. Mori, Scr. Metall. 9, 631 (1975).

${ }^{20}$ J. Narayan and Y. T. Zhu, Appl. Phys. Lett. 92, 151908 (2008)

${ }^{21}$ X. L. Wu, J. Narayan, and Y. T. Zhu, Appl. Phys. Lett. 93, 031910 (2008)

${ }^{22}$ Y. T. Zhu, J. Narayan, J. P. Hirth, S. Mahajan, X. L. Wu, and X. Z. Liao, Acta Mater. 57, 3763 (2009)

${ }^{23}$ J. Y. Huang, Y. T. Zhu, H. G. Jiang, and T. C. Lowe, Acta Mater. 49, 1497 (2001).

${ }^{24}$ J. P. Hirth and J. Lothe, Theory of Dislocations, 2nd ed. (Krieger, Malabar, FL, 1992), p. 117.

${ }^{25}$ X. Z. Liao, S. G. Srinivasan, Y. H. Zhao, M. I. Baskes, Y. T. Zhu, F. Zhou, E. J. Lavernia, and H. Xu, Appl. Phys. Lett. 84, 3564 (2004). 